\title{
A Planar IrO Multichannel Stimulating Electrode for Use in Neural Prostheses
}

\author{
S. J. TANGHE, K. NAJAFI and K. D. WISE
}

Center for Integrated Sensors and Circuits, Department of Electrical Engineering and Computer Science, University of Michigan, Ann Arbor, MI 48109-2122 (U.S.A.)

\begin{abstract}
This paper describes a multisite stimulating probe capable of the precise delivery of sophisticated charge waveforms to neural structures on a chronic basis. The fabrication process for passive probes requires only four masks and is very high yield. Activated iridium oxide stimulating sites are shown to be compatible with the delivery of over $3 \mathrm{mC} / \mathrm{cm}^{2} /$ phase in biphasic charge-balanced operation. In order to minimize the number of external probe leads, on-chip circuitry has been defined to allow per-channel current control at the 8-bit level using serial input data and a total of five external leads.
\end{abstract}

\section{Introduction}

Electrical stimulation is a promising technique for the restoration of a variety of physiological functions, including controllable limb movement, hearing and, perhaps (longer-term), vision [1]. However, a major problem limiting the widespread application of functional electrical stimulation has been the lack of stimulating electrodes that can be used for long-term precise multipoint stimulation of muscle and nerve cell populations. Stimulating electrodes for use in depth have typically been made using needle microelectrodes, which lack appropriate controls over site size and shape and cannot be satisfactorily formed in arrays. The use of electrode arrays is of critical importance in stimulating nerve bundles and in allowing precise current/charge patterns to be built up as the sum of many spatially distributed sites to achieve enhanced selectivity. The development of suitable stimulating arrays requires the ability to form many independently controlled sites in a structure inducing minimal tissue damage and the use of site materials which are capable of delivering very high charge densities to the tissue on a chronic basis. This paper reports the development of a high-yield, IC-compatible fabrication process for multielectrode stimulating probes, the characterization of iridium and activated iridium as a site material at high charge densities, and the design of system electronics, including on-chip interface circuitry, to improve system reliability and minimize interconnects to the external world.

\section{Probe Structure and Fabrication}

Figure 1 shows the overall structure of the multielectrode stimulating probe. A micromachined silicon substrate supports an array of thinfilm conductors which are insulated above and below by deposited dielectric films and which connect stimulating sites near the tip of the probe with control circuitry at the rear of the substrate. In order to fabricate these passive structures, a deep boron diffusion is first used to define the intended substrate. Stress-relieved dielectrics of silicon nitride and silicon dioxide are next deposited, followed by interconnects of either tantalum or polysilicon. Refractory silicides might also be used. After patterning the interconnects, the upper dielectrics are deposited and openings are defined for the stimulating sites and the bonding pads. The stimulating site material is deposited at this point and defined using lift-off. The site material is thus self-aligned to the dielectric openings. Following final definition of the field dielectrics, the wafer is placed in an anisotropic silicon etch (ethylenediamine pyrocatechol) and the wafer is dissolved, leaving only the desired probes. This

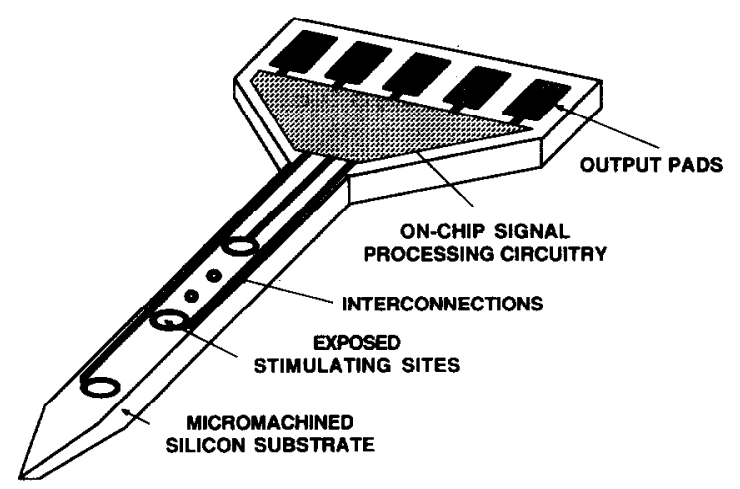

Fig. 1. Structure of an active multielectrode stimulating probe.

(C) Elsevier Sequoia/Printed in The Netherlands 


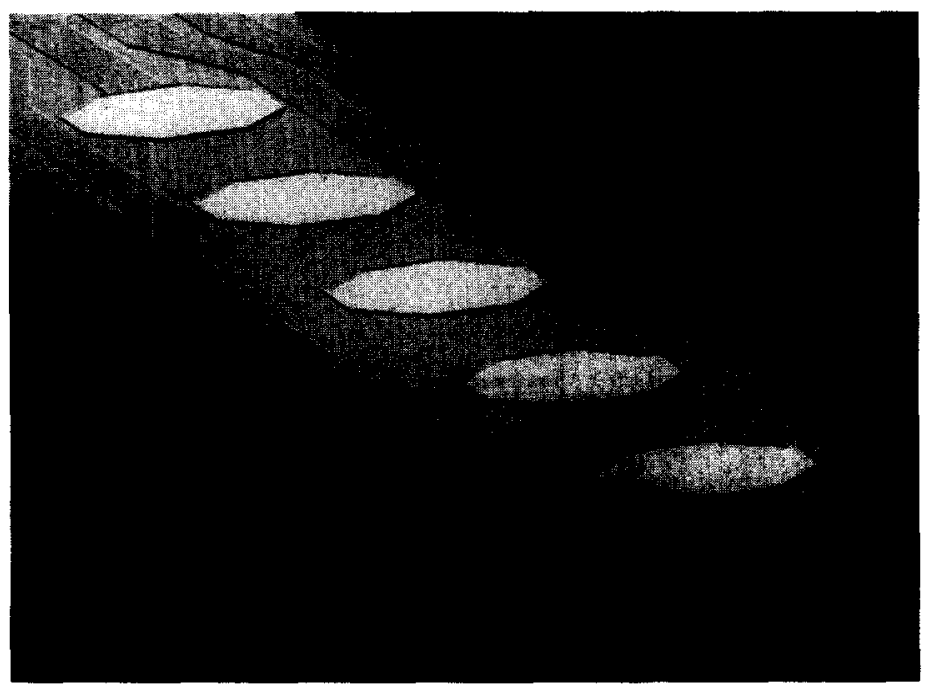

Fig. 2. SEM view of the tip of a multisite probe. The site areas here are $8000 \mu \mathrm{m}^{2}$, with an intersite separation of $200 \mu \mathrm{m}$.

final etch is entirely self-stopping and is very high yield. The process is single-sided and requires only four masks. All pattern definition except for wafer dissolution is performed using dry etching. Figure 2 shows an SEM view of a five-site passive probe using this process. The substrate here is $15 \mu \mathrm{m}$ thick and the site areas are $8000 \mu \mathrm{m}^{2}$.

\section{Selection and Activation of the Stimulating Sites}

It is important to select a material for the stimulating sites which is biocompatible, compatible with the overall probe-formation process, and capable of delivering high charge densities to the tissue. It is the injected charge which has been shown to stimulate the neuronal action potential, and this charge must be delivered without inducing gas evolution, gross $\mathrm{pH}$ changes, or metal corrosion near the site. Iridium has been shown to meet all these requirements. It is a noble metal and the densest and most corrosion resistant known. It can be deposited by sputtering and defined using lift-off. Iridium oxide is porous and can assume several different oxidation states [2], which gives it a very high charge capacity as well as the ability to transfer large amounts of charge to aqueous solution. The oxide can be produced using anodic activation, thermal oxidation, or by reactive sputtering. The iridium oxide films used in this work were produced by the first of these techniques. The sites were electrically activated by repeated voltage cycling in carbonate-buffered saline to form a hydrous iridium oxide [3]. The voltage sweep rate and voltage limits are important in setting the charge capacity and in avoiding stress-related cracking of the resulting oxide films.

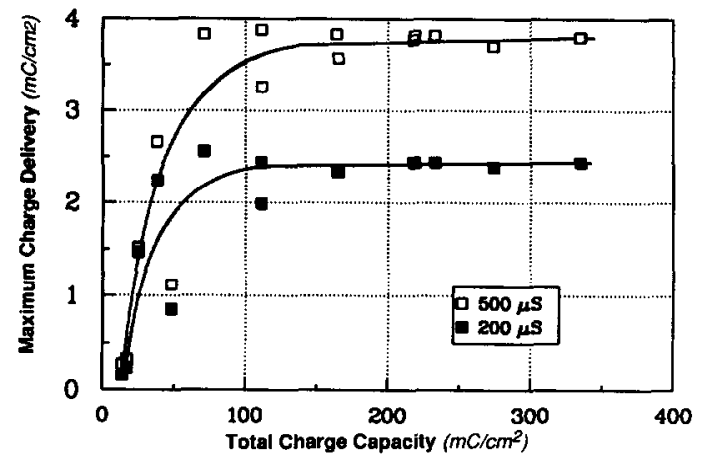

Fig. 3. Charge delivery of activated iridium oxide films as a function of total film charge capacity. The data are for four $100 \mu \mathrm{m}^{2}$ sites activated in saline at $2.1 \mathrm{~V} / \mathrm{s}$ between $-0.9 \mathrm{~V}$ and $+1.2 \mathrm{~V}$ (vs. SCE). The pulse durations are 500 and $200 \mu \mathrm{s}$.

Figure 3 shows the per-phase charge which can be delivered to solution via charge-balanced current pulses as a function of the charge capacity of the film. This capability for high charge delivery on the part of iridium oxide exceeds that for metals such as $\mathrm{Au}$ and Pt by well over an order of magnitude. Activated stimulating sites have been subjected to over one million charge-balanced biphasic current pulses ( $100 \mu \mathrm{A}$ for $200 \mu \mathrm{s} /$ phase or about $250 \mu \mathrm{C} / \mathrm{cm}^{2} /$ phase) without any significant shift in the electrical characteristics of the site or any physical deterioration of the iridium oxide or adjacent dielectric films.

\section{Organization of the System Electronics}

The successful long-term application of these multielectrode stimulating probes depends to a 


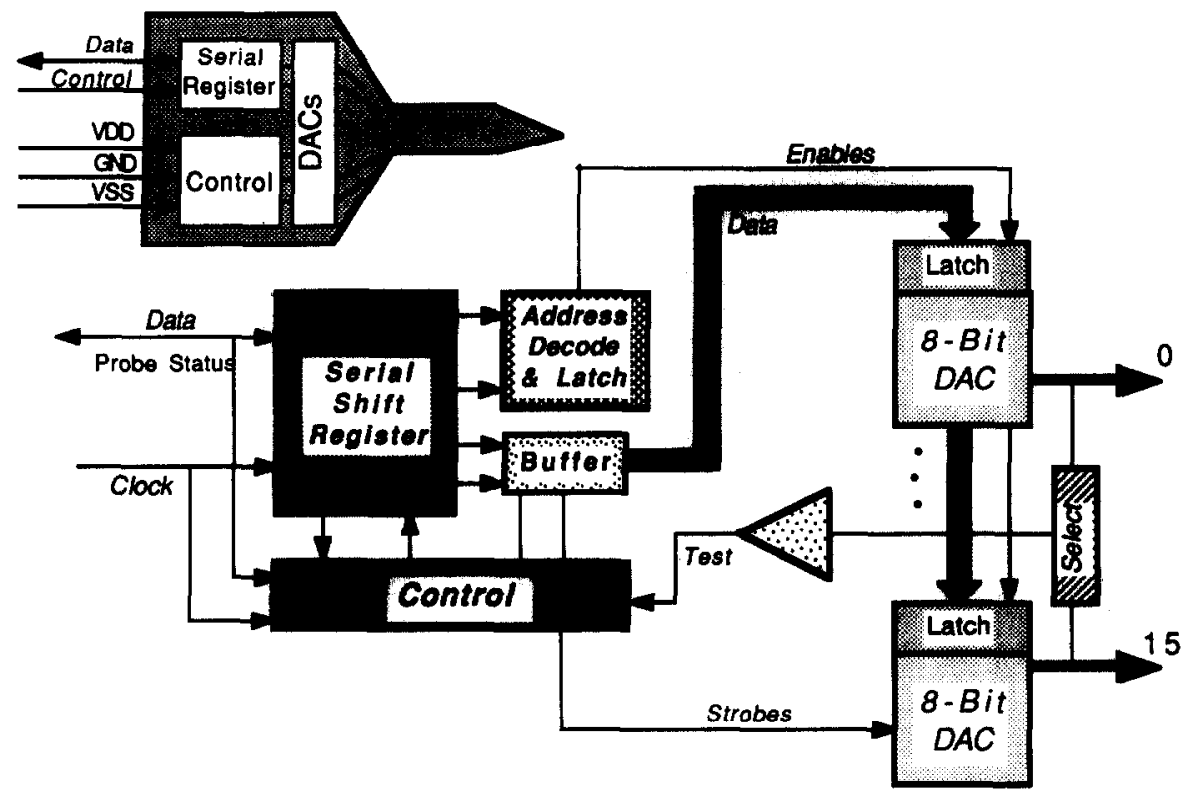

Fig. 4. Block diagram of the electronics for a I6-site stimulating probe with independent per-channel control of current level and self-test capability.

large extent on the successful integration of onchip electronics with the stimulating sites to minimize the number of external probe leads, reduce tethering, and improve overall system reliability. Figure 4 shows a block diagram of the on-chip circuitry required for a 16-site stimulating probe, while Fig. 5 shows the signaling protocols and data word structure for the system. The probe accepts serial data inputs which are used to control per-channel stimulating currents with an amplitude resolution of eight bits ( 0 to $\pm 256 \mu \mathrm{A}$ biphasic $\pm 2 \mu \mathrm{A}$ ). All timing is controlled off-chip, and all channels can be active in parallel. To initiate a given pulse, 14 bits of data are entered serially using the data and clock lines. The data word includes four bits of channel address, eight bits to control the biphasic current amplitude, and two mode bits for remote testing and probe calibration. Two additional time slots are used per pulse: one for a strobe bit to control data entry

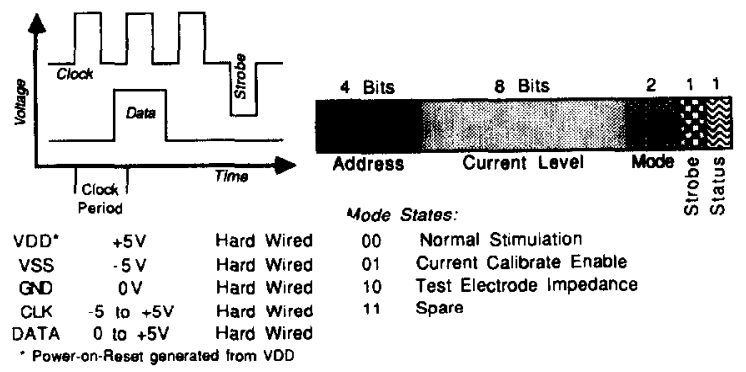

Fig. 5. Signaling protocols (left) and data word structure (right) for an active 16-channel stimulating probe. and one during which the probe can signal the host if any on-board problems are detected. Extensive on-chip safety checks are included to prevent accidental or unwanted stimulation, including a per-channel time-out feature to reset a pulse in the event it exceeds a predetermined width. The active probe requires only five external leads: power (VDD, VSS and GND) plus DATA and CLOCK. The on-chip probe electronics have been designed using a $\pm 5 \mathrm{~V} 3 \mu \mathrm{m}$ bulk p-well double-poly single-metal epi-CMOS process. The full circuitry requires approximately 5500 transistors in a layout area of $6.8 \mathrm{~mm}^{2}$ and is now in the final layout stage of development.

\section{Conclusions}

A process for producing passive multielectrode stimulating probes for use in neural prostheses has been described. The process produces a very high yield and is compatible with probes of arbitrary two-dimensional shape which are biocompatible, minimally invasive, and capable of delivering charge densities in excess of $3 \mathrm{mC} / \mathrm{cm}^{2} /$ phase. Active electronics have also been defined to allow the probes to function as part of a high-performance system, capable of delivering coded pulse patterns to the tissue at a rapid rate and requiring only five external probe leads. The multielectrode passive stimulating probes with activated iridium oxide films have been successfully used to stimulate auditory pathways. Stimulation by passing current between different sites on a single probe 
has provided highly selective stimulation, with electrophysiological thresholds similar to or lower than those for conventional single or multipolar wire electrodes. The ability to deliver precisely controlled currents over a wide dynamic range and with small individual site areas should enable these devices to be used in a variety of prosthetic devices where long-term multipoint selective stimulation of biological tissue is required.

\section{Acknowledgements}

This work was supported by the National Institutes of Health under contract NIH-NINCDS-86-
09. The authors wish to express their appreciation to Drs F. T. Hambrecht and W. Heetderks of the Neural Prosthesis Program, NIH, for their support and encouragement in this work.

\section{References}

1 F. T. Hambrecht and J. Reswick (eds.), Functional Electrical Stimulation: Application in Neutral Prostheses, Marcel Dekker, New York, 1977.

2 R. Kotz, N. Neff and S. Stucki, Anodic iridium oxide films, J. Electrochem. Soc., 131 (1984) 72-77.

3 L. Robblee, J. Leftko and S. Brummer, Activated iridium: an clectrode suitable for reversible charge injection into saline solutions, J. Electrochem. Soc., 130 (1983) 731-733. 